\title{
Editorial on: Topical Collection on InSight Mission to Mars
}

\author{
W.B. Banerdt ${ }^{1} \cdot$ C.T. Russell ${ }^{2}$
}

Published online: 9 October 2017

(C) Springer Science+Business Media B.V. 2017

Over the past twelve decades seismology has been one of our most important tools in understanding the Earth and its deep interior. So it is not surprising that the value of seismology in the exploration of extraterrestrial planets has been recognized since the dawn of the space age. The very first spacecraft that were sent by the United States in the early 1960s to land on the surface of another planet (Rangers 3-5, designed to survive impact on the Moon) carried rugged seismometers, and exquisitely sensitive seismic instruments were a key component of the scientific payloads brought to the Moon by the Apollo program. When Vikings 1 and 2 executed the first landings on Mars in 1976 they also carried seismometers (which, for various reasons, did not return much useful information). These were relatively modest instruments that were envisioned to be the vanguard of a more extensive network of seismometers that would probe the deep interior of the planet, revealing its structure and processes.

So it is somewhat surprising that in the 40 years since the Viking landings there has not been another seismometer placed on the surface of any planet beyond the Earth. This is not for lack of trying. In the early 1990s a Mars Network Mission with a dozen seismic stations was a solid component of NASA's strategic plan for Mars exploration leading up to sample return. Later in the decade this mission was replaced in the planning process by MESUR (Mars Environmental SURvey) at NASA, supplemented by the proposed MarsNet, and later the InterMarsNet, projects at ESA. Together, these ambitious projects were to place 16-20 long-lived landers with seismometers and meteorological instrument packages on the surface of Mars, using 4-5 launches in the 2001-2003 time period. Mars Pathfinder was originally conceived as a development project for producing a low-cost lander for MESUR. But in 1996 cost pressures, combined with a new emphasis on the search for life on Mars, led to the delay and eventual cancellation of these seismic network missions. The Russian

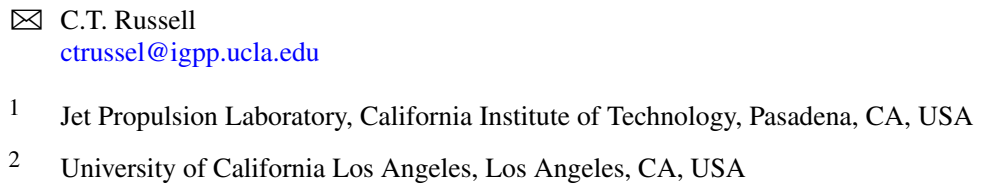


Mars'96 mission carried two French and two Russian seismometers, but was stopped short of Mars by an upper stage failure shortly after launch.

Hopes for a seismic network were revived in the late 1990s and early 2000s with NetLander, a more modest network of four landers. This CNES-led project was initially to be carried by ESA's Mars Express, and later was part of a NASA-CNES collaboration centered on Mars sample return, slated for launch in 2007. But NetLander did not survive the collapse of the Mars Sample Return project in 2003, and a plan to fly a NetLander seismometer on ESA's ExoMars lander was short-lived.

Much of the difficulty with getting seismometers to Mars can be traced to the relatively high cost of these missions. In addition to the technical challenges that seismometers place on a Mars lander (e.g., extended life, direct access to the surface, low mechanical noise, high data volumes), the sheer number of landers involved in a seismic network of 4-20 stations constitutes an enormous financial barrier. This led a group of planetary geophysicists in the mid-2000s to conceive of a different approach to Mars seismology. Instead of assuming "conventional" seismic arrival time techniques, which require multiple widely-separated seismometers to triangulate event locations and determine their times of occurrence, a single extremely sensitive, three-component, broad-band seismometer would utilize a variety of sophisticated, but existing, single-station analysis techniques to accomplish the scientific objectives of delineating the major divisions of the deep Martian interior in order to advance our understanding of planetary formation and evolution. This led to the GEMS (Geophysical and Environmental Monitoring Station) proposal to NASA's Mars Scout Program in 2006, and finally to the successful GEMS proposal to NASA's Discovery Program in 2010. Because another mission within NASA had previously adopted the GEMS acronym, the mission was renamed InSight, for Interior exploration using Seismic Investigations, Geodesy and Heat Transport.

The InSight payload is focused on probing the deep interior of Mars using geophysical techniques, with a seismometer and heat flow probe along with a radio transponder that allows precision tracking of Mars' rotation. In addition, it has a set of instruments to monitor the environment (pressure, wind, air and ground temperature, magnetic field) in order to better interpret the extremely sensitive seismic data.

This special volume contains 22 papers that describe investigations that were carried out largely prior to the originally-planned launch in March of 2016, in order to guide the design of the mission and its instruments, support the landing on Mars and enhance the value of the data that would be acquired thereafter. (Unfortunately, a late failure of the vacuum enclosure for the seismometer necessitated a 26-month delay of the launch; thus a subsequent volume is planned that will include mission and instrument description papers.) The overall evaluation of potential landing sites was led by M. Golombek who describes the considerations in choosing the site. This is followed by papers led by first-authors R.A. Beyer, R.L. Fergason and N.E. Putzig on specific aspects of the chosen landing site from remote sensing. The properties of the top several meters of the soil are important to the InSight mission because the heat flow experiment depends on a 'mole' that drives itself 3-5 meters into the soil to emplace a system of thermal sensors. The production of the regolith and its properties are described in two papers led by N.H. Warner and P. Delage, and the mole and its physical interaction with the soil are described in papers led by J. Poganski and by J.P. Marshall. The thermal effects of the lander and the environment on the on the heat-flow determination in the subsurface is described by M.A. Siegler et al. and P. Morgan et al., respectively. S. Kedar and co-authors describe a planned experiment to determine regolith elastic properties from the mole's seismic signatures. B. Knapmeyer-Endrun et al. describe Rayleigh wave modeling and inversion for landing site structure. D. Mimoun et al. present an overall model of 
the seismic noise InSight should find on Mars, whereas N. Murdoch et al. specifically cover modeling of seismic wind noise and pressure noise in a pair of articles and N.A. Teanby et al. discuss the coupling of wind noise with the seismometer through the regolith. B. Kenda et al. discuss the seismic effects of dust devils. J. Stevanovic et al. treat the seismic detection of bolide air bursts, and R.F. Garcia et al. model the infrasound signals emitted by meteor impacts. E. Bozdağ et al. examine simulations of seismic wave propagation, while S. Ceylan et al. discuss the creation of synthetic seismograms for initial Mars models. This volume closes with an article on the planned products of the Mars structure service by M. Panning and others.

This special volume on the InSight mission results from the efforts of many people; from the authors of the papers listed above, to the engineers and managers who have supported the program. A very special thank you goes to the referees of this volume who are the quality control team providing guidance to the authors in the preparation of their articles. Helping on this very important task were: Don Banfield, Ross Beyer, Ebru Bozdağ, Bernardo Caicedo, Lynn Carter, Douglas Dreger, Caleb Fassett, Francesca Ferri, Matt Golombek, Bob Grimm, Matthias Grott, James W. Head, III, Shaopeng Huang, Bob Hutt, Heiner Igel, Walter Kiefer, Randy Kirk, Sharon Kedar, Norbert Koemle, Mark Lemmon, Qinya Liu, Ralph Lorenz, David Mimoun, Francis Nimmo, Andi Petculescu, Thomas Platz, Karol Seweryn, Matthew Siegler, Tilman Spohn, Sarah Sutton, Toshiro Tanimoto, Chad Trabant, Victor Tsai, Ashwin Vasavada, Christos Vrettos, Rudolf Widmer-Schnidrig, Jean-Pierre Williams, Yingyje Yang, Yingcai Zheng, and Richard Zurek. The editors are also grateful for the strong support by Springer-Nature and by Marjorie Sowmendran of the University of California Los Angeles. Their assistance has been indispensable in completing the editorial process. 